\title{
An Approach to Library Automation Problems
}

\begin{abstract}
The introduction of automation systems into libraries requires considerable planning. The relative independence of the various internal operations should be recognized and evaluated. Independent operations may be automated in an order which gives the maximum gain for the least cost. The interdependent operations should be automated in an order which follows through the logical sequence of operations. Information used in an automated system should be critically evaluated to see whether it is relevant to the process, and every effort should be made to see that the automated and manual parts are in balance.
\end{abstract}

W ITH THE INCREASED PRESSURE On libraries to expand their collections it is inevitable that modifications of traditional library procedures soon are necessary. One of these is automation. If used intelligently automation can be of substantial benefit to libraries, but unfortunately in many cases the benefits promised have not materialized. Some feel that insufficient knowledge exists in libraries as to how to merge automated processes with or into non-automated processes. This paper is an attempt to bridge this gap.

\section{The Sequence of Automation}

The first problem facing an investigating team, apart from the availability of funds, is deciding in what sequence a library automation program should take place.

In a theoretical situation, a library could be considered a series of independent operations or functions. To some extent this is true; for instance, the circulation function is largely inde-

Dr. Jackson is Systems Coordinator in the Library of Pennsylvania State University. pendent of the acquisitions function, although obviously there is some relationship between the two in the sense that in a large library the activities in both functions probably would likely be considerable. This interpretation is misleading, however, as both functions are mutually correlated with library size and there may in fact be very little true correlation between the two functions. The important thing to consider is that an increase in activity in the acquisitions function would not necessarily result in an increase in the activity within the circulation function.

If the assumption of independence is made, the problem of selecting the automation sequence becomes simple. In each case an estimated or projected cost may be assigned to the automation of an individual function. Probably to all intents and purposes a safe assumption would be that the cost involved would be linearly proportional to the time taken to automate that particular function.

In addition to the cost aspect there is the profit aspect. This is much more difficult to assign as in many cases the profits must be expressed in an intangible form. Every effort should be made, 
however, to derive some form of profit figure which, even if it is in an intangible form, gives an indication of the relative worth of automating a particular function.

When a time (or cost) figure has been obtained and an estimate has been made of the profits to be accrued from automating each function, the function which should be automated first is the one giving the maximum expected profit for the least amount of time spent in automating $i t$. Subsequently the various functions should be ordered in a sequence which corresponds to the ratios of the various functions, arranged in order of decreasing numeric size.

On this basis, then, the decision on whether to automate the circulation or the acquisitions departments would depend on the relative advantages to be obtained from an automation project, weighted by the time it would take to get the automation project finished.

While it may be argued that an ideal library system consists of a series of independent functions, in actual practice this is seldom the case. For instance, in most libraries, the cataloging function is closely related to the acquisition function. To a lesser degree an automated circulation department could be expected to be tied in with an automated catalog department in the sense that the output from cataloging is the input to the circulation department. The problem is to apply automation where it will cause as little disruption as possible to the rest of the system. On the other hand, once a function is automated it should be as near as possible to its final form. Thus, as a general rule, an automated function should not have to be altered at a later date as other automated functions are introduced. This restriction is not, however, all-inclusive, and in each case the cost of adding the information at a later date must be weighed against the cost of keeping the information in the system, always bearing in mind the fact that excessive information at any stage tends to reduce the speed with which the information can be handled. Thus, if the acquisitions and cataloging functions are both to be automated it may be cheaper to add the catalog information at a relatively late stage in the system (it may have to be added in a revised form anyway).

This paper would propose that the solution to the sequential problem is to start automation at the beginning of a series of operations and to work gradually through the system in order. Thus the general automation sequence would be acquisitions and then cataloging. In this manner, while recognizing the sequential nature of the operations, the overall system is divided into a series of subsystems which are successively optimized. Where subsystem B follows subsystem A, if B is optimized and then A is optimized, the over-all results will not necessarily be the same as if $\mathrm{A}$ is first optimized and then B. In all probability, however, the latter sequence, with A optimized before B, will be more effective than the former.

From a theoretical point of view, also, the combined effects of the optimization of $\mathrm{A}$ and of the optimization of $\mathrm{B}$ will not necessarily be equal to the sum of the separate effects. This is because of the interaction effect, which may be negative, decreasing the over-all effects. On the other hand, the interaction may be positive with the result that the overall performance is increased.

In a practical situation in a library automation project, however, the individual separate positive effects will tend to be small, because of the effects of the many disruptions inevitably introduced into the rest of the system, whereas the interaction effects are likely to be large and positive as a result of the elimination of these disturbances. For instance, if the acquisitions function is separately automated, there is likely to be a considerable disruption of the cataloging 
function. Similarly, if cataloging is separately automated, some interference with the routine in acquisitions would be expected. If both functions were automated, however, it would be expected that the disruptions would disappear and the combined positive effects would be substantial.

\section{The Automation Process}

With a decision having been made to start an automation project with the acquisitions function or department, data may be captured at its source, that is, when a book request enters the department. On the other hand, just because information is available does not necessarily mean that it should enter the system. If it will not be used it should not enter the automated part of the system. For instance, information may be written on a book request form to indicate who should be notified when the book is cataloged and shelved. It may be the practice within the library to return a copy of the original order to the request originator. In this case there is no need to transfer the information from the order form to the automated system, to store and manipulate it within the system as the book is processed, and then when the information is required, to consult the original request form only and ignore the rest.

Each bit of information should be evaluated critically to see whether or not it would be used if it entered the system. For instance, if an acquisitions librarian had an order form in his hand and he required some information, it would be pointless for him to consult a computer-produced listing when all he had to do is to look at the form. Thus, for this particular application, the listing would possibly be just as useful if it were printed with less information. If the listing is used to obtain some information, however, it is probable that the listing should have contained the additional information in the first place.
Computer-produced records should contain minimum information necessary. Failure to observe this point is the biggest weakness in many automated library systems. Systems designers sometimes seem to pride themselves on the amount of information contained in their records. What such practice overlooks is that it is a very costly process to use a computer as a printer. With the high acquisitions rate a list can become very long and if unnecessary multiple lines are produced for each entry the cost can be prohibitive. In fact, the problem of getting adequate information from the computer onto hard copy listings may well be one of the biggest problems to be faced in library automation today.

Because of the importance of this problem it is well to dwell upon it at some length. Probably, under normal searching conditions, the alphabetic file is the most commonly used of all order files. With manual access, information is added slip by slip and removed in the same manner. The inactive slips are not handled at all. This is not true for a computer-produced listing. Each time a listing is produced the whole listing, from $\mathrm{A}$ to $\mathrm{Z}$, must be produced, although perhaps less than 10 per cent of the file has changed since the last listing. The remaining 90 per cent has remained unchanged, with some entries remaining unchanged through as many as ten listings. The list must be produced for a small percentage of the entire file with the result that the change cost per entry becomes excessively high.

An alternate solution is to produce a supplement. From a work study point of view this virtually doubles the number of files that must be searched. In addition, if an entry has changed from one status condition, to another, the old entry may have been found in the main file while the more recent and correct entry is ignored. Also, in spite of claims frequently made to the contrary, it is probable that the very existence of a 
supplement causes a substantial increase in the time taken to find the required information. With both a listing and a supplement available the tendency is to look in both places, even though the probability of finding an entry in the supplement is comparatively small.

A further'strategy is to have the longer listing produced less frequently; it can always be shown that in any situation there is an optimum time interval for producing a listing. This interval is based upon the cost of producing the listing and the cost of purchasing duplicates, or of any mistakes caused by the delay in appearance of successive listings. This strategy appears to be unsatisfactory from a librarian's viewpoint because, notwithstanding theory to the contrary, when a book is not found interested parties are left in the dark as to the current status of the book order, or the whole system grinds to a halt while the offending entry is traced down.

Alternatively, the entry may be shortened until it consists of only one line of computer output. This has the effect of reducing the cost of the listing so that the interval between successive listings may be reduced. While the problem of producing the entire listing from A to $\mathrm{Z}$ remains, the situation is somewhat less critical as the list is considerably shorter.

The librarian still appears to be on the losing end of the deal, however, as he must now contend with abbreviated entries. With a limit of about 130 characters per line this is inevitable. If the author entry is lengthened, the title is abbreviated. If the title is lengthened, the author must be shortened.

The word "appears" is used above because it may well be that the reason for the apparent inadequacies of the list is not the form of the lists themselves but how they are used. Initially, for instance, it may be assumed that whatever the form of the list or the printout, the in- formation supplied to the vendor on the book purchase order must be complete in the sense that is sufficient to enable the vendor to identify the book. The primary purpose of information transfer from the library to the vendor is thus accomplished. Since it may also be assumed that the original form on which the book request was written has been retained, this information is also available to the library. Thus, irrespective of how the listings are used, information is not lost to the system. Also, in an automated system, the computer fulfills the function of correlating the incoming information with existing information, a function for which it is ideally suited. Although some correlation may, of necessity, be done manually, it should be kept to a minimum. The listings should be of the form that enable the majority of the noncomputerized activities to be completed efficiently. If this can be accomplished with abbreviated entries or partial information such a listing is adequate. In the relatively rare cases in which additional information is required it may be extracted from the original request cards.

\section{The Automation Balance}

Although much of the previous section has been devoted to acquisitions procedures, the same principles apply to other library functions. In every case a balance must be obtained between the automated and the manual parts of the system. This does not necessarily mean weighing the quantity of work performed by each part in balance with maximum over-all work efficiency.

In the accounting function, for instance, it does not necessarily follow that all the work should be handled by the computer. The accounting may be so complex that in order for the computer to obtain the information necessary for the calculations, complicated manual procedures must be initiated. 
This in essence defeats the purpose of automation. In such a case the solution probably is to simplify the ovếr-all accounting procedures. In many cases, however, it may be much eheaper to retain some aspects of the manual system, possibly redesigned to merge effciently with the various automated data processing operations.

A circulation control systen is a case in point. From a theoretical point of view an automated circulation system is very simple. Information from a borrower and from a book is fed into a memory. If the book is returned before its expiration date the item is erased from the memory. If it is not returned a message is given that the book is overdue. Side operations may or may not include inventory listings. In its most elemental form an automated system consists of a keypunch and a sorter, but a duplicator, interpreter, and accounting machine may also be included. In this case the memory would consist of a card file. In the more complex case the memory consists of magnetic discs under the control of a computer with some form of input and output terminals.

It is undoubtedly true that the manual circulation systems used in most libraries are very efficient. The problem arises out of the sheer bulk of transactions carried out within the circulation department. Because of staff difficulties, for example, it may no longer bè possible to maintain and follow up overdue notifications adequately. The solution in such a situation may not necessarily be to introduce a simple automated system (keypunches, sorters, etc.), as the manual system may be transferred to the automated system with the added expense of the machine rental being very high in relationship to the advantages gained.
If a computer system is introduced, the machine (i.e., console) rental cost may be small but the rental of the memory banks and the computer costs may be considerable. The advantages gained from the automation would have to be substantial before such a system could be implemented. Possibly the optimum arrangement would be a combination of the efficient aspects of the manual system, one or two simple data processing machines (i.e., keypunches), and the use of a computer on a batch basis for some of the more time-consuming operations.

\section{Conclusions}

In an evaluation of a library for automation purposes the various functions within the library must be delineated and classified with respect to each other. Some activities will emerge as being independent of the others. These may be automated on the basis of obtaining the greatest profit from the least amount of effort. The independent activities may then be arranged in a descending sequence of expected profit per unit of automation time. The operations are then automated in the order of their respective expected profit figures.

During the process a critical evaluation must be made of the information in the system to see if it is relevant to the particular activity being considered. No superfluous information should be carried, especially with a computer, where printing costs may become prohibitive. The nature of library information lists is such that extensive study must be made of them at an early stage in the automation process. In addition, considerable care must be taken that the automated and manual parts of the system are in balance with excessive emphasis being placed on neither. 